\title{
Aprendendo Contexto para Adaptar Processo
}

\author{
Juliana do Espirito Santo Carvalho ${ }^{1}$, Flavia Maria Santoro ${ }^{1}$, Kate Revoredo ${ }^{1}$, \\ Vanessa Tavares Nunes ${ }^{2}$ \\ ${ }^{1}$ Departamento de Informática Aplicada - Universidade Federal do Estado do Rio de \\ Janeiro (UNIRIO) - Rio de Janeiro - RJ - Brasil \\ ${ }^{2}$ COPPE/PESC - Universidade Federal do Rio de Janeiro (UFRJ)- Rio de Janeiro - RJ - \\ Brasil. \\ \{juliana.carvalho, flavia.santoro, katerevoredo\} @uniriotec.br, \\ vanunesdcos.ufrj.br
}

\begin{abstract}
Dynamic adaptation aims to make a business process applicable to a particular situation during its life cycle. Adapting requires experience, and involves knowledge about various aspects of business. We argue for the application of adaptation rules, considering the context of a particular process instance. However, a context-based adaptation environment should go beyond, and learn from its decisions, as well as continuously identify new unforeseen situations. The goal of this paper is to present a methodology to infer the need to update situations and adaptation rules, suggesting changes to them. An application scenario is presented to discuss the proposal.
\end{abstract}

Resumo. Adaptação dinâmica torna um processo de negócio aplicável a uma determinada situação, em qualquer momento do seu ciclo de vida. Adaptação requer experiência, e envolve conhecimento sobre vários aspectos do negócio. Assim, propomos a aplicação das regras de adaptação, considerando o contexto de cada instância de um processo. No entanto, um ambiente de adaptação baseada em contexto deve ir além, e aprender a partir de suas decisões, bem como identificar continuamente novas situações imprevistas. $O$ objetivo deste trabalho é apresentar uma metodologia para inferir a necessidade de atualizar as situações e regras de adaptação, sugerindo alterações. Um cenário de aplicação é apresentado para discutir a proposta.

\section{Introdução}

Flexibilidade em processos de negócio é uma demanda atual nas organizações, a qual está relacionada com a capacidade de um processo de se adaptar, mantendo a sua estabilidade e alinhamento com as políticas, cultura e objetivos organizacionais [Nunes et al 2011]. Adaptação dinâmica de um processo de negócio é a ação de personalizar o processo para torná-lo aplicável a uma situação particular, a qualquer momento do seu ciclo de vida. No entanto, adaptar requer experiência e envolve conhecimento sobre vários aspectos do negócio, tais como, meio ambiente, pessoas, tecnologias utilizadas e a própria organização em si, além de aspectos externos a ela [Brézillon and Pomerol 1999]. Mecanismos devem ser definidos para possibilitar a adaptação em ambientes de execução de processos, incluindo a definição de regras de adaptação que levem em consideração o contexto de uma determinada instância do processo [Nunes et al 2011b]. 
Contexto é definido como uma informação útil para a execução das atividades e para as interações que ocorrem em um processo de trabalho [Hallerbach et al 2007]. Sua importância está no fato de que fornece uma relação forte de causa-efeito entre as exigências de flexibilidade e os impactos sobre a adaptação de processos [Nunes et al 2011]. O conhecimento contextual está nas experiências compartilhadas entre participantes, artefatos, atividades coletivas e individuais, condições e fatos que acontecem durante a execução de um processo [Nunes et al 2012].

O contexto da ocorrência de uma atividade em um processo de negócio (chamado aqui de Situação) deve ser identificado a partir de um conjunto de elementos contextuais (EC). Situação é formalmente definida como: Situação $A=\{E C 1=v 1, E C 2$ $=\mathrm{v} 2, \ldots, \mathrm{ECn}=\mathrm{vn}\}$, onde $\mathrm{ECi}, \forall \mathrm{i}$ com $1 \leq \mathrm{i} \leq \mathrm{n}$, são elementos contextuais, vi, o valor que ECi assume em um dado momento, e, Situação A é a definição da circunstância causada por esta condição. A ocorrência de uma situação pode exigir uma adaptação do processo. Por outro lado, a adaptação com base no contexto requer decisões sobre que regras de adaptação aplicar [Mattos et al 2012].

Desta forma, regras para adaptação dinâmica de um processo podem ser definidas conforme as situações mapeadas, ou seja, toda regra de adaptação de processo está vinculada a alguma situação identificada. Já regras de adaptação possuem o formato Se (condição) - Então (ação), onde a condição é a definição de contexto (Situação) já identificada e a ação é a atitude, a decisão a ser tomada de forma a adaptar o processo dinamicamente em tempo de execução objetivando mantê-lo alinhado aos objetivos traçados para ele [Nunes 2011].

Por exemplo, considerando o cenário de controle de tráfego aéreo, especificamente o processo de aeronaves decolando, alguns elementos contextuais podem interferir neste processo, tais como, o espaço aéreo, condições meteorológicas e de aeronaves. Eles são especificados junto com seus possíveis valores, e situações que capturam os potenciais problemas para o fluxo normal do processo são modeladas, por exemplo, Situação $\mathrm{A}=\{$ Espaço Aéreo = "operacional com restrição" e condição de tempo $=$ "desfavorável" $\}$. Uma regra de adaptação é criada para resolver essa situação.

Em um dia com muita neblina e com muitas decolagens e pousos previstos, caracterizando presença da situação $\mathrm{A}$, a regra de adaptação manda que a decolagem seja adiada por um período de uma hora. Entretanto, após uma hora prevista, a neblina continua intensa, o que não permite que a decolagem seja realizada (objetivo não atingido). Observa-se que ao longo do tempo tem ocorrido casos com essa mesma descrição de situação, com a mesma tomada de decisão para contorná-la e com os mesmos resultados. Em outras palavras, observa-se que na maioria das vezes que a situação A ocorre o resultado do processo não tem sido atingido.

Partindo do cenário descrito aqui, argumenta-se que é fundamental para um ambiente de adaptação dinâmica de processos baseado em contexto, ir além, e aprender com decisões tomadas, bem como, identificar novas situações não previstas. Para tal, é importante manter uma base histórica contendo informações de contexto associadas às instâncias de atividades executadas e experiências sobre as adaptações de processo realizadas. Armazenar o contexto permitirá que atores e sistemas identifiquem instâncias similares, possibilitando aprender em cada ciclo de adaptação e inferir novas 
regras e padrões, trazendo mais dinamismo à execução do processo e alinhamento aos objetivos organizacionais.

O objetivo deste artigo é apresentar um mecanismo computacional que infere a necessidade de atualização de definições de contexto e regras de adaptação e, quando necessário, sugere evoluções para estas visando uma adaptação dinâmica contextualizada de processos de negócio independente do domínio. Na Seção 2 é apresentada a proposta do artigo, na Seção 3 um cenário de aplicação. É realizada uma breve discussão a cerca de adaptação dinâmica sensível ao contexto na Seção 4. Os trabalhos relacionados são apresentados na Seção 5 e as conclusões na Seção 6.

\section{Proposta para Atualização das situações e regras de adaptação}

Neste artigo dois tipos de atualizações sobre o repositório de contexto são definidas (i) atualização da base de regras de adaptação e (ii) atualização da base de definições de contexto. A primeira tem por objetivo manter as regras de adaptação condizentes com os objetivos traçados para o processo e a segunda mantém as definições de contexto factíveis de acontecerem durante a execução do processo. Para tal, armazenar-se-á uma série de informações sobre cada instância de cada atividade do processo já executada. Além de armazenar a atividade, serão armazenadas também informações sobre as possíveis situações (definições de contexto) identificadas na atividade, as regras de adaptação utilizadas para adaptar a instância do processo dadas as situações, e a indicação se o processo atingiu os seus objetivos ou não.

Examinar o histórico de instâncias de processo é significativo, uma vez que situações e regras de adaptação são inicialmente definidas considerando a experiência de analistas de negócios. Entretanto, depois de várias execuções do processo, devido ao grande volume de dados gerados, torna-se cada vez mais difícil analisar manualmente se as situações e regras de adaptação definidas permanecem consistentes e relevantes para o processo.

Por outro lado, o processo de descoberta de conhecimento em banco de dados (DCBD) [Han and Kamber 2006], onde a mineração de dados é a uma das etapas, tem por objetivo aprender automaticamente conhecimento a partir de uma base de dados. Um formato de entrada amplamente utilizado em DCBD é um banco de transações. Transações são dados correlatos que estão em uma mesma tupla no banco de dados [Han and Kamber 2006]. Um algoritmo que implementa essa técnica procura identificar relações entre os itens desse conjunto de dados, que são descritas em forma de regras, denominadas regras de associação, do tipo "Se X então $Y$ ", ou "X -> Y", onde X e Y são conjuntos de itens, denominados itemsets, e $\mathrm{X} \cap \mathrm{Y}=\varnothing$. $\mathrm{O}$ objetivo de um algoritmo para descoberta de regras de associação é identificar todas aquelas que tenham suporte (Sup) e confiança (Conf) maiores do que os valores mínimos estipulados. Suporte é um número mínimo de ocorrências que contém na mesma transação $\mathrm{X}$ e $\mathrm{Y}$, e confiança é o percentual de transações que satisfazem determinadas regras, ou seja, é a frequência com que $Y$ aparece nas transações que contêm $X$ [Witten et al 2011]. O algoritmo mais usado na implementação de regras de associação é o algoritmo Apriori [Witten et al 2011], que faz uma varredura no conjunto de dados procurando por subconjuntos que tenham relacionamentos que sejam frequentes, em outras palavras, se 
um itemset (conjunto de um ou mais itens) é frequente, então todos os seus subconjuntos também são frequentes.

Neste trabalho, aplicamos o algoritmo Apriori [Witten et al 2011] para fornecer algumas sugestões para a necessidade de definir novas situações ou regras de associação. Para isso, é feita uma análise estatística do log de instâncias de atividades, identificando os casos em que o objetivo do processo não foi alcançado. Se a porcentagem de casos que não tiveram seus resultados iguais aos objetivos do processo for maior do que um determinado valor determinado pelos especialistas, então existe uma necessidade de determinar qual é a razão potencial para o problema. Esta análise será feita para cada atividade do processo.

O fluxo de execução do mecanismo proposto é ilustrado na Figura 1. Com a mineração de dados através do algoritmo Apriori, será possível:

- Sugerir novas Situações

- Indicar a necessidade de modificações nas regras de adaptação.

- Indicar a necessidade de busca de novos elementos contextuais.

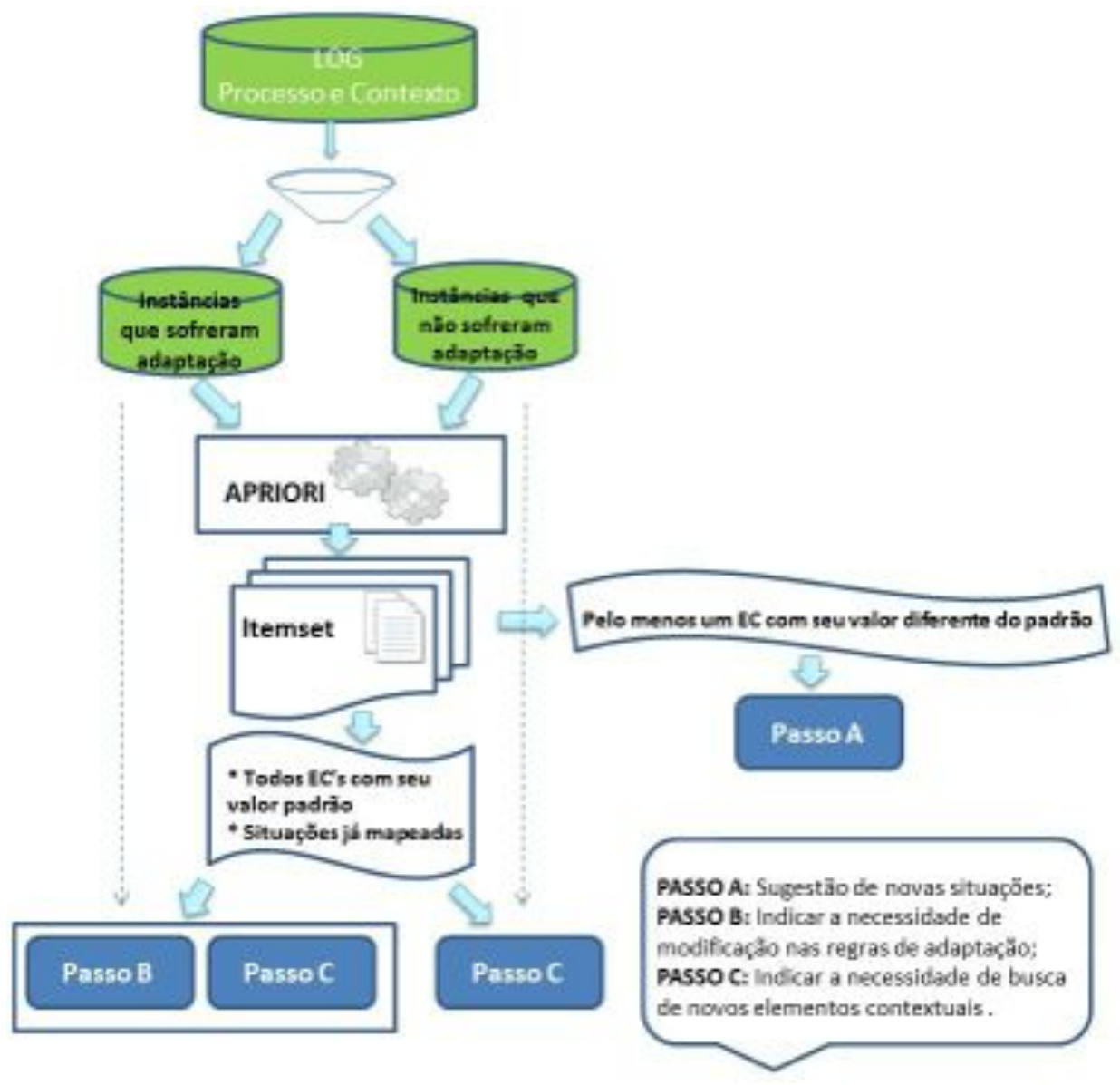

Figura 1 - Fluxo da Proposta considerando os 3 cenários.

A extração de dados deve ser realizada continuamente ao longo do tempo, em um intervalo definido pelos gestores de processo. O objetivo é garantir que o modelo de contexto esteja sempre atualizado, através do exame da relevância das situações pré- 
definidas e, consequentemente, as regras de adaptação que devem ser usadas para resolvê-las, e também os elementos contextuais que são combinados para defini-las. Deve ser aplicada em duas condições diferentes: instâncias adaptadas e instâncias que não precisaram ser adaptadas. Depois disso, em ambas as condições, a análise dos itemsets gerados pelo Apriori deve ser feita para identificar itemsets (i) que tenham pelo menos um elemento contextual com um valor não-padrão e (ii) que não fazem parte de uma situação já definida. Estes itemsets são fortes candidatos para explicar uma nova situação.

\section{A. Passo A - Sugestão de Novas Situações.}

A primeira condição a ser analisada é em instâncias em que ocorreu uma situação, a regra de adaptação correspondente foi aplicada, mas o objetivo do processo não foi atingido. Uma razão possível para isso pode estar na definição da situação.

A segunda condição é exatamente o contrário: não houve necessidade de adaptação em tempo de execução (nenhuma situação foi identificada, portanto, nenhuma regra foi aplicada para adaptar), no entanto, percebe-se que os resultados de muitas instâncias não são compatíveis com os objetivos do processo. A causa potencial para este problema pode ser que novas situações podem estar interferindo no processo, mas elas não foram identificadas ainda.

Em ambas as condições, os especialistas podem identificar novos padrões do conjunto de dados, através dos itemsets sugeridos pelo Apriori entre os elementos contextuais atuais. Estes novos padrões poderiam sugerir novas situações que são reconhecidas a partir do rearranjo de elementos contextuais já identificados.

\section{B. Passo B - Indicar a necessidade de modificações nas regras de adaptação.}

Se uma nova situação não foi descoberta na primeira condição descrita no Passo A, um dos problemas pode ser a regra de adaptação correspondente aplicada em tempo de execução. Por alguma razão, ela não ajuda mais a instância do processo a alcançar suas metas.

Portanto, a recomendação é que especialistas analisem a regra de adaptação aplicada, e desenhem novas estratégias para lidar com os problemas provocados pela ocorrência de tal situação. A fim de tentar confirmar essa possibilidade e até mesmo poder indicar qual regra especificamente está com possibilidade de desatualização, o Apriori é executado novamente para cada regra de adaptação, ou seja, para cada grupo formado por instâncias que utilizaram uma dada regra.

\section{Passo C - Indicar a necessidade de busca de novos elementos contextuais.}

Ainda na primeira condição descrita no Passo A, o problema pode estar nos elementos contextuais identificados para o processo. Ou seja, podem existir novos elementos contextuais que caracterizam e influenciam o processo e que não foram previamente identificados.

Quando a segunda condição do Passo A ocorre, e o algoritmo aplicado não descobre novas situações, talvez isso signifique que existam outros elementos contextuais relevantes ainda não conhecidos que possam estar interferindo no processo. Anastassiu (2012) propõe um método para a identificação de elementos contextuais de processos de negócios internos à organização. Então, neste momento, sugerimos a execução desse 
método, a fim de descobrir outros elementos contextuais relevantes. Eles serão então monitorados e armazenados, e a partir daí, novas situações poderão ser descobertas.

O método aqui proposto deve ser executado periodicamente para capturar e analisar cada um dos três cenários prováveis que podem ter ocorrido entre as instâncias do processo dentro do log. No Passo A, é possível sugerir novas situações para atualizar o modelo de contexto, enquanto que nos Passos B e C, recomenda-se instruções para o analista verificar o modelo. Na seção seguinte, apresentamos um cenário de aplicação, onde a proposta foi aplicada para obter uma visibilidade mais precisa e completa sobre o processo e suas instâncias.

\section{Cenário de Aplicação}

Nosso cenário de aplicação é sobre o domínio de controle de espaço aéreo. O Controle de Tráfego Aéreo é um serviço prestado por controladores que orientam e monitoram aeronaves, para garantir um fluxo de tráfego seguro e ordenado. O processo em questão é o de decolagem de aeronave, apresentado em [Mattos 2012].

Nesse processo cinco fatores podem caracterizar algum tipo de perigo para a sua execução: Homem, Meio, Máquina, Gerenciamento e Missão. Para cada fator de perigo, foram mapeados elementos contextuais e seus possíveis valores. Na Tabela 1, são apresentados alguns elementos contextuais, separados por tipo de perigo, seus possíveis valores e seu valor padrão, destacado em negrito.

\section{Tabela 1 - Elementos Contextuais.}

\begin{tabular}{|c|c|}
\hline \multicolumn{2}{|r|}{ Tipo de Perigo: Homem } \\
\hline $\begin{array}{l}\text { Controlador de Tráfego } \\
\text { Aéreo }\end{array}$ & $\begin{array}{l}\text { Descrição da condição do controlador de tráfego aéreo no instante } \\
\text { monitorado. Valores: atuação padrão, atuação interrompida. }\end{array}$ \\
\hline \multicolumn{2}{|r|}{ Tipo Perigo: Máquina } \\
\hline Aeronave & $\begin{array}{l}\text { Descrição da condição da aeronave no instante monitorado. Valores: } \\
\text { operacional, não operacional. }\end{array}$ \\
\hline \multicolumn{2}{|r|}{ Tipo Perigo: Meio } \\
\hline Condição Meteorológica & $\begin{array}{l}\text { Descrição da condição meteorológica no instante monitorado. Valores: } \\
\text { favorável, desfavorável, impeditiva. }\end{array}$ \\
\hline Pista & $\begin{array}{l}\text { Descrição da condição da pista no instante monitorado. Valores: } \\
\text { operacional, não operacional. }\end{array}$ \\
\hline Espaço Aéreo & $\begin{array}{l}\text { Descrição da condição do espaço aéreo no instante monitorado. } \\
\text { Valores: operacional, não operacional, operacional com restrições. }\end{array}$ \\
\hline
\end{tabular}

Para esses elementos contextuais foram identificadas as seguintes definições de contexto (situação):

- Decolagem Improvável $(\mathrm{DI})=$ \{Espaço Aéreo = "Operacional com Restrições", Condição Meteorológica $=$ "Desfavorável" $\}$;

- Decolagem Extremamente Improvável $(\mathrm{DEI})=\{$ Espaço Aéreo = "Não Operacional", Aeronave $=$ "Operacional" 
É importante notar que todas as situações têm pelo menos um elemento contextual assumindo um valor diferente do valor padrão, o que indica que ocorreu uma condição anormal. Assim, uma adaptação do processo é justificada.

Cada Situação tem uma regra de adaptação mapeada para ela. Sua definição não é relevante para o nosso mecanismo proposto. Neste cenário de aplicação, o log de execução tem ocorrências relativas à atividade "Transmitir informações e parâmetros de voo", que inclui as informações e os parâmetros necessários para a atividade de decolagem (conforme a Figura 2). Vinte e dois casos foram considerados, sete alcançaram a meta de decolagem, enquanto quinze não o fizeram.

\begin{tabular}{|c|c|c|c|c|c|c|c|}
\hline \multirow{2}{*}{$\begin{array}{c}\text { HOMEM } \\
\text { Runway operator }\end{array}$} & \multirow{2}{*}{$\begin{array}{c}\text { MÁQUINA } \\
\text { Aircraft }\end{array}$} & \multicolumn{3}{|c|}{ MEIO } & \multirow{2}{*}{ SITUAÇÃO } & \multirow{2}{*}{$\begin{array}{c}\text { REGRA DE } \\
\text { ADAPTAÇÃOO }\end{array}$} & \multirow{2}{*}{ OBJETIVO } \\
\hline & & Weather condition & Runway & Airspace & & & \\
\hline Operacao_padrao & Operacional & Desfavoravel & Operacional & $\begin{array}{c}\text { Operacional_com_ } \\
\text { restricoes }\end{array}$ & DI & 1 & Falso \\
\hline Operacao_padrao & Operacional & Desfavoravel & Operacional & $\begin{array}{c}\text { Operacional_com_ } \\
\text { restricoes }\end{array}$ & DI & 1 & Falso \\
\hline Operacao_padrao & Operacional & Desfavoravel & Operacional & $\begin{array}{c}\text { Operacional_com_ } \\
\text { restricoes }\end{array}$ & DI & 1 & Verdadeiro \\
\hline Operacao_padrao & Nao_Operacional & Favoravel & Nao_Operacional & Operacional & Nenhuma & Nenhuma & Falso \\
\hline Operacao_padrao & Operacional & Favoravel & Operacional & Operacional & Nenhuma & Nenhuma & Verdadeiro \\
\hline Operacao_padrao & Operacional & Favoravel & Operacional & Operacional & Nenhuma & Nenhuma & Verdadeiro \\
\hline Operacao_padrao & Nao_Operacional & Favoravel & Nao_Operacional & Operacional & Nenhuma & Nenhuma & Falso \\
\hline Operacao_padrao & Nao_Operacional & Favoravel & Nao_Operacional & Operacional & Nenhuma & Nenhuma & Falso \\
\hline Operacao_Interrompida & Operacional & Favoravel & Operacional & Nao_Operacional & DEI & 2 & Falso \\
\hline Operacao_Interrompida & Operacional & Favoravel & Operacional & Nao_Operacional & DEI & 2 & Falso \\
\hline Operacao_padrao & Operacional & Favoravel & Operacional & Nao_Operacional & DEI & 2 & Verdadeiro \\
\hline Operacao_Interrompida & Operacional & Favoravel & Operacional & Nao_Operacional & DEI & 2 & Falso \\
\hline Operacao_padrao & Operacional & Favoravel & Operacional & Nao_Operacional & DEI & 2 & Verdadeiro \\
\hline Operacao_padrao & Operacional & Favoravel & Operacional & Operacional & Nenhuma & Nenhuma & Verdadeiro \\
\hline Operacao_padrao & Nao_Operacional & Favoravel & Nao_Operacional & Operacional & Nenhuma & Nenhuma & Falso \\
\hline Operacao_padrao & Operacional & Favoravel & Operacional & Operacional & Nenhuma & Nenhuma & Verdadeiro \\
\hline Operacao_padrao & Operacional & Favoravel & Operacional & Operacional & Nenhuma & Nenhuma & Falso \\
\hline Operacao_padrao & Operacional & Favoravel & Operacional & Operacional & Nenhuma & Nenhuma & Falso \\
\hline Operacao_padrao & Operacional & Favoravel & Operacional & Operacional & Nenhuma & Nenhuma & Falso \\
\hline Operacao_Interrompida & Operacional & Favoravel & Operacional & Nao_Operacional & DEI & 2 & Falso \\
\hline Operacao_padrao & Operacional & Favoravel & Operacional & Operacional & Nenhuma & Nenhuma & Falso \\
\hline Operacao_padrao & Nao_Operacional & Favoravel & Nao_Operacional & Operacional & Nenhuma & Nenhuma & Falso \\
\hline
\end{tabular}

Figura 2 - Log após a execução de 22 instâncias.

A Figura 3 mostra os agrupamentos possíveis das informações do log para a execução do algoritmo, quatro possíveis execuções para as adaptadas e duas para as não adaptadas. $\mathrm{O}$ algoritmo foi executado para instâncias adaptadas, e em duas das quatro possibilidades: para cada regra, analisando apenas as instâncias que não atingiram o objetivo (Possibilidade 2) e para todas as regras sem distinguir se as instâncias atingiram o objetivo ou não (Possibilidade 3). O valor mínimo de suporte foi de $60 \%$ e o valor mínimo de confiança de $80 \%$, valores escolhidos por trazerem resultados que representem mais da metade das instâncias, além disso, foram estabelecidos não muito próximos aos $100 \%$ já que a quantidade de instâncias é pequena. O toolbox WEKA [Machine Learning Group 2012] foi utilizado. Todos os itemsets considerados aqui superaram o suporte.

Para a possibilidade 2, o algoritmo foi executado duas vezes. Primeiramente apenas para as duas instâncias que aplicaram a regra 1 e que não atingiram o objetivo. A execução do algoritmo gerou cinco itemsets de tamanho 1 , dez de tamanho 2, dez de 
tamanho 3, cinco de tamanho 4 e um de tamanho 5. Todos os itemsets que envolvem os elementos contextuais "condição meteorológica" e "espaço aéreo" os apresentam com seus valores fora do valor default, "Desfavorável" e "Operacional com Restrições", respectivamente. Através da execução do algoritmo, não foi possível identificar novas situações, uma vez que todos os itemsets que apresentam elementos contextuais fora do valor default possuem elementos com os mesmos valores que caracterizam a situação DI. Isto sugere que os especialistas devem reavaliar a regra 1, porque esta regra não poderá mais adaptar o processo corretamente, quando está sob a interferência da situação DI.

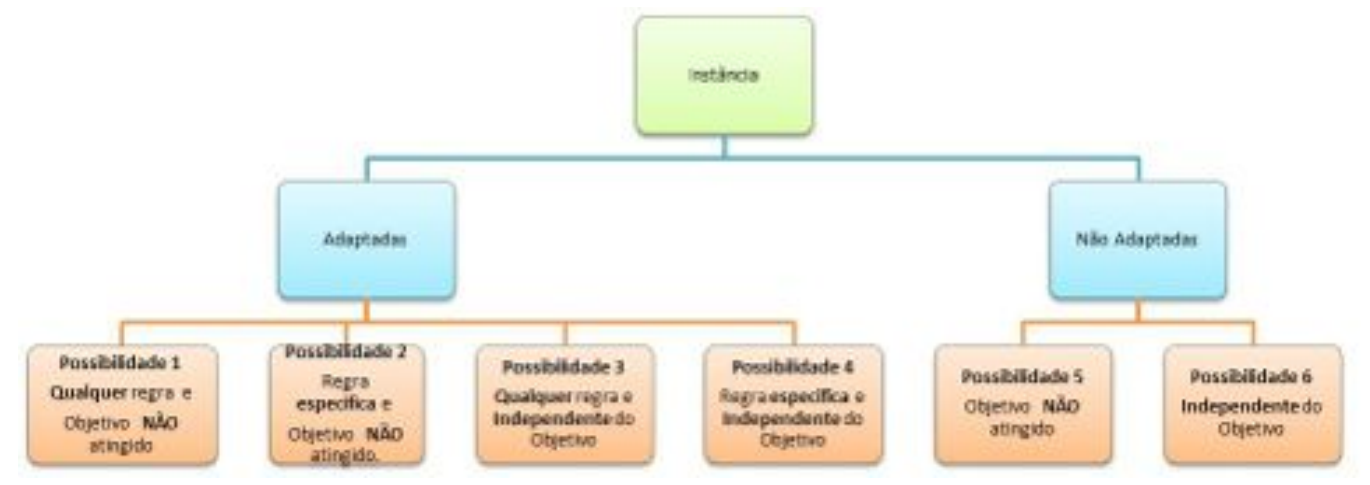

Figura 3 - Condições possíveis para execução do Apriori

Em seguida, para as quatro instâncias que aplicaram a regra 2 e que não atingiram o objetivo. A execução do algoritmo gerou cinco itemsets de tamanho 1, dez de tamanho 2, dez de tamanho 3, cinco de tamanho 4 e um de tamanho 5. Todos os itemsets que envolvem os elementos contextuais "controlador de trafego aéreo" e "espaço aéreo" os apresentam com seus valores fora do valor default, "Atuação Interrompida" e "Não Operacional", respectivamente. Desta forma, através da execução do algoritmo foi possível identificar novas situações, uma vez que todos os itemsets que envolviam o elemento contextual "controlador de trafego aéreo" o apresentava em seu valor não default e aliado a isso se têm o fato que a situação DEI, que demandou a utilização da regra 2, não possui em sua definição esse elemento contextual. Então, todo itemset que envolva ele juntamente com "espaço aéreo" é uma potencial nova situação, cabendo aos especialistas optar por ela ou não.

Para a possibilidade 3, o algoritmo foi executado para as nove instâncias que demandaram adaptação, independente de terem atingido o objetivo. A execução do algoritmo gerou cinco itemsets de tamanho 1, oito de tamanho 2, cinco de tamanho 3 e um de tamanho 4. O único elemento contextual apresentado fora do seu valor default foi "espaço aéreo" que aparece como "Não operacional". Dos elementos contextuais que caracterizam a situação DI nenhum deles aparece com o valor configurado para essa situação, o que não nos permite inferir nada sobre o que pode estar acontecendo com as instâncias que executaram a regra 1 e não atingiram o objetivo, ainda que esse seja o resultado encontrado em mais de $60 \%$ das instâncias que sofreram interferência dessa situação. Sobre a situação DEI, também nada se pode inferir, uma vez que dos elementos contextuais apresentados pelo Apriori aqueles que configuram essa situação são apresentados exatamente como descritos na situação. Esses resultados nos levam a concluir que esse cenário de execução do algoritmo não é indicado, uma vez que se trata 
de um cenário muito generalista não avaliando casos específicos, dessa forma, não tornando possível identificar as possíveis causas de algumas instâncias não terem atingindo o objetivo mapeado para o processo.

\section{Discussão}

Uma aplicação da nossa metodologia é no Sistema de Gestão de Contexto (GCAdapt) apresentado em [Nunes et al 2012]. Este sistema atua como um servidor central apoiando o gerenciamento do ciclo de vida de contexto (captura, armazenamento, raciocínio e recuperação de contexto) para adaptação dinâmica de processo. O GCAdapt é composto por quatro componentes: Agregador, Mediador, Tratador e Atuador, além de uma série de mecanismos de captura de elementos contextuais e de mecanismos de implementação de processos, que variam de acordo com o ambiente da organização, o tipo de elementos contextuais considerados e o Sistema de Informação Sensível ao Processo utilizado.

O módulo mantenedor gerencia Definição Situacional e Regras de Adaptação no Repositório de Regras de Contexto. O módulo de Definição Situacional contém o modelo de elementos contextuais e instâncias do processo associado à caracterização do contexto durante a execução. O módulo de Regras de adaptação mantém regras de adaptação do processo com base em situações de contexto. A proposta aqui apresentada poderá atuar nesses dois módulos, uma vez que eles possuem, respectivamente, a função de identificar novas situações (reconfigurar uma situação existente e/ ou excluir uma situação que não seja mais válida para a organização) e identificar, definir novas regras de adaptação, alterar e excluir regras existentes. Assim, através do método proposto será possível manter atualizados o repositório de definições de contexto e o repositório de regras de adaptação. O primeiro mantém as definições de situação, assim como o modelo conceitual de contexto. O segundo mantém as regras de adaptação indicando para qual situação a regra foi definida.

\section{Trabalhos Relacionados}

O trabalho apresentado em [Bucchiaroneet al 2011] propõe um framework de apoio à adaptação dinâmica de processo sensível ao contexto baseado na instância de execução do processo e no histórico de adaptação. Em vez de procurar adaptações recorrentes, eles propõem olhar para as necessidades de adaptação recorrentes (ou seja, instâncias do processo com a violação em um mesmo contexto de restrição e configuração do sistema). Com base na análise dos casos adaptados, automaticamente constroem e classificam variantes de evolução corretiva que podem lidar com o contexto problemático. Ao mesmo tempo, tenta-se identificar variantes de evolução preventiva através da construção de variantes do processo que podem evitar a necessidade de adaptação. O framework evolutivo proposto por eles é composto por três fases: Fase de execução, fase de análise e fase de evolução. Apesar de apoiar a adaptação dinâmica de processo sensível ao contexto com base na instância de execução, essa abordagem não foca na manutenção e atualização das definições de contexto nem das variantes do processo.

O Process Variants by Options (Provop) proposto em [Hallerbach et al 2007] apresenta uma solução mais flexível para gestão de variantes em todo o ciclo de vida do 
processo através do uso de Opções, que são o agrupamento de operações de mudanças. Essas operações podem ser uma inserção, deleção, modificação ou apenas reordenação de elementos do processo. Assim, permite a configuração dinâmica das variantes de processo com base no contexto do processo, isto é, as operações de mudança necessárias para criar a variante do respectivo processo são dinamicamente selecionadas com base na informação contextual. Os autores argumentam que variantes do processo normalmente têm de ser definidas e mantidas em modelos separados do modelo do processo principal, resultando em dados redundantes uma vez que os modelos de variantes possuem entre si a maioria de suas partes semelhantes. Com isso, os modelos de variantes do processo degeneram ao longo do tempo, uma vez que atualizações aplicadas em um único modelo de variantes não levará em consideração a relação com as outras variantes. Apesar de trabalhar com a definição de contexto (como os elementos contextuais) e definindo as relações entre eles (como a situação), este trabalho não propõe a evolução dos dados relacionados a esses dois conceitos. É proposta a evolução de variante, no entanto, ao contrário deste trabalho, essas variantes são definidas como um conjunto de elementos do modelo de processo e não como regras de adaptação.

PAIS (Sistemas de Informação Sensíveis a Processo), "sistema de software que gerencia e executa os processos operacionais envolvendo pessoas, aplicações e / ou fontes com base em modelos de processos", é uma abordagem tecnológica de apoio (semi) automatizado à adaptação dinâmica de processo [Dumas et al 2005]. Entre os PAIS existentes, destacam-se [Schonenberg et al 2008]: YAWL [van der Aalst e Hofstede 2005], FLOWer [Dumas et al 2005], Declare [Pesic; van der Aalst 2006] e ADEPT [Reichert et al 2003], cada um apoiando a flexibilidade de uma maneira diferente. PAIS permite mudanças sem recodificar as partes do sistema, mas nenhum deles suporta a adaptação em termos de adequação para o negócio (nível semântico), mas apenas em um nível sintático assegurando que qualquer adaptação não vai de alguma forma corromper o processo. Além disso, as análises e consequentes adaptações sugeridas nesses PAIS são feitas manualmente. As decisões sobre a adaptação não são registradas, e nem uma análise sobre elas é feita.

Mineração de processo é realizada em logs de processos executados objetivando extrair conhecimento desse conjunto de execuções reais. Através da mineração de processo é possível descobrir o modelo do processo através da ordem de relação entre as várias tarefas. Além disso, é possível verificar se um modelo gráfico mapeado para um processo é realmente condizente com o que é tipicamente executado. É possível também realizar uma análise de performance com o intuito de melhorar o modelo do processo e suas propriedades. Alguns trabalhos têm utilizado a mineração de dados como apoio ou como forma de enriquecimento da mineração de processos [Rozinat and van der Aalst 2006] [Saravanan and Rama Sree 2011]. Esses trabalhos utilizam a mineração de dados objetivando analisar ou classificar os resultados obtidos pela mineração de processos e não como forma de manter atualizadas as bases de situações e regras de adaptação objetivando auxiliar a adaptação dinâmica contextualizada de processos.

Nossa proposta difere da que também visa a adaptação de processos com base em dados históricos [Bucchiaroneet al 2011], uma vez que usamos a técnica de regras de associação pelo algoritmo Apriori para sugerir mudanças no contexto (situações, regras de adaptação e elementos contextuais), e não para modificá-lo em tempo de execução ou rever o modelo. Nossa abordagem procura identificar a razão para a 
divergência entre os objetivos do processo destinados e seus resultados reais, sugerindo possíveis soluções, ao contrário das abordagens PAIS que abordam o problema em um nível sintático (estrutura do modelo). A abordagem que está mais próxima da nossa é Provop [Hallerbach et al 2007], no entanto, sugerem mudanças nos elementos do modelo de processo e não no contexto, que é o nosso foco.

\section{Conclusões}

Um modelo de processo de negócio convencional pode atender às necessidades dos clientes em um determinado contexto e não em outro [Saidani et al 2007]. Observa-se que há aspectos que influenciam na execução de processos apoiando a ideia de que não se pode prever tudo o que acontece durante a sua execução, surgindo novas situações e alterando o desempenho dos processos [Nunes et al 2011].

Nesse cenário, esse trabalho propõe um mecanismo que irá inferir a necessidade de modificação nas definições de contexto e regras de adaptação. Esse mecanismo deve ser executado em ambientes que armazenem logs de execuções do processo. Com esse mecanismo será possível manter as definições de contexto atualizadas e factíveis e as regras de adaptação que permitem que o resultado da execução do processo esteja sempre alinhado com os objetivos traçados para o processo.

Como trabalho futuro, pretende-se implementar esta proposta em um processo real, através de estudos de caso em organizações com o objetivo de ampliar os resultados obtidos.

\section{Referências}

ANASTASSIU, M. Um Método para Identificação de Elementos Contextuais que Impactam em Processos de Negócio. Dissertação de Mestrado, Programa de PósGraduação em Informática, UNIRIO, Rio de Janeiro, Brasil, 2012.

BRÉZILLON, P., \& POMEROL, J. C. Contextual knowledge sharing and cooperation in intelligent assistant systems. Le Travail Humain, 223-246, 1999.

BUCCHIARONE, A., MARCONI, A., PISTORE, M., \& SIRBU. A context-aware framework for business processes evolution. In: Enterprise Distributed Object Computing Conference Workshops (EDOCW), 2011 15th IEEE International. IEEE, 2011. p. 146-154.

DA CUNHA MATTOS, T., SANTORO, F. M., REVOREDO, K., \& NUNES, V. T. Formalizing the situation of a business process activity. In Computer Supported Cooperative Work in Design (CSCWD), 2012 IEEE 16th International Conference on (pp. 128-134). IEEE, 2012.

DUMAS, M., VAN DER AALST, W. M., \& TER HOFSTEDE, A. H. Process-aware information systems: bridging people and software through process technology. Wiley-Interscience 2005.

HALLERBACH, A., BAUER, T., \& REICHERT, M. Managing Process Variants in the Process Lifecycle. 2008.

HAN, J., KAMBER, M. Data Mining: Concepts and Techniques. San Francisco: Morgen Kaufmann Publishers. 2006. 2nd edition. ISBN 978-1-55860-901-3. 
MACHINE LEARNING GROUP AT UNIVERSITY OF WAIKATO, "WEKA". Disponível em: http://www.cs.waikato.ac.nz/ml/weka Acessado em: 28 de dezembro de 2012.

MATTOS T. Um modelo para formalização de contexto em processos de negócio. Dissertação de Mestrado, Programa de Pós-Graduação em Informática, UNIRIO, Rio de Janeiro, Brasil, 2012

NUNES, V. T. Adaptação dinâmica de processos: Uma abordagem orientada a contexto. PHD Qualifier Exam, COPPE, UFRJ, Rio de Janeiro, Brasil, 2011.

NUNES, V. T., WERNER, C. M. L., \& SANTORO, F. M. Mediating process adaptation through a goal-oriented context-aware approach. InComputer Supported Cooperative Work in Design (CSCWD), 2012 IEEE 16th International Conference on (pp. 160-167). IEEE 2012.

NUNES, V. T.; WERNER, C.M.L.; SANTORO, F. M. Dynamic process adaptation: A context-aware approach. In: Proceedings of The 15th International Conference on Computer Supported Cooperative Work in Design. New Jersey: IEEE Press, 2011 a. v.1. p. 97-104.

NUNES, V., WERNER, C., SANTORO, F. AND BRÉZILLON, P. Contextualizing dynamic process adaptation. 7th International Workshop on Modeling and Reasoning in Context, Karlsruhe, Germany, September 26-30, Springer Verlag, LNCS, 2011b.

PESIC, M., \& VAN DER AALST, W. M. A declarative approach for flexible business processes management. In: Business Process Management Workshops. Springer Berlin Heidelberg, 2006. p. 169-180.

REICHERT, M., RINDERLE, S., \& DADAM, P. Adept workflow management system. In Business Process Management (pp. 370-379). Springer Berlin Heidelberg 2003.

ROZINAT, A., \& VAN DER AALST, W. M. Decision mining in ProM. In:Business Process Management. Springer Berlin Heidelberg, 2006. p. 420-425.

SAIDANI, O., \& NURCAN, S. Towards context aware business process modelling. In 8th Workshop on Business Process Modeling, Development, and Support (BPMDS'07), June 11-12, 2007, Trondheim, Norway.

SARAVANAN MS, RAMA SREE RJ,(2011) Application of Mining Algorithms using ProM and Weka Tools. IJCST Vol. 2, Issue 3, Setembro 2011.

SCHONENBERG, H., MANS, R., RUSSELL, N., MULYAR, N., \& VAN DER AALST, W. Process flexibility: A survey of contemporary approaches. In: Advances in Enterprise Engineering I. Springer Berlin Heidelberg, 2008. p. 16-30.

VAN DER AALST, W. M., \& TER HOFSTEDE, A. H. YAWL: yet another workflow language. Information systems, 30(4), 245-275, 2005.

WITTEN, I. H., FRANK, E., HALL, M. A.: Data Mining: Practical Machine Learning Tools and Techniques. 3rd ed. :Morgan Kaufmann, 2011, ISBN: 978-0-12-374856-0. 